\title{
Edema Cerebral, CTCAE
}

National Cancer Institute

\section{Source}

National Cancer Institute. Edema Cerebral, CT CAE. NCI Thesaurus. Code C143431.

A disorder characterized by swelling due to an excessive accumulation of fluid in the brain. 